\title{
Photochemical processes in flavo-enzymes as a probe for active site dynamics: TrmFO of Thermus thermophilus
}

\author{
Bo Zhuang ${ }^{1}\left[\right.$ Lipsa $\mathrm{Nag}^{1} \cdot$ Pierre Sournia $^{1} \cdot$ Anastasia Croitoru $^{1}{ }^{1} \cdot$ Rivo Ramodiharilafy $^{1}{ }^{(1)}$.

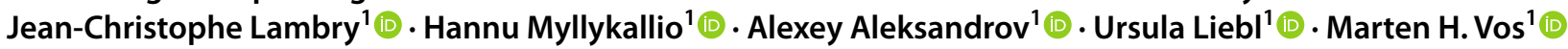

Received: 26 March 2021 / Accepted: 3 May 2021

(c) The Author(s), under exclusive licence to European Photochemistry Association, European Society for Photobiology 2021

\begin{abstract}
Quenching of flavin fluorescence by electron transfer from neighboring aromatic residues is ubiquitous in flavoproteins. Apart from constituting a functional process in specific light-active systems, time-resolved spectral characterization of the process can more generally be employed as a probe for the active site configuration and dynamics. In the C51A variant of the bacterial RNA-transforming flavoenzyme TrmFO from the bacterium Thermus thermophilus, fluorescence is very shortlived ( $\sim 1 \mathrm{ps})$, and close-by Tyr343 is known to act as the main quencher, as confirmed here by the very similar dynamics observed in protein variants with modified other potential quenchers, Trp283 and Trp214. When Tyr343 is modified to redox-inactive phenylalanine, slower and highly multiphasic kinetics are observed on the picosecond-nanosecond timescale, reflecting heterogeneous electron donor-acceptor configurations. We demonstrate that Trp214, which is located on a potentially functional flexible loop, contributes to electron donor quenching in this variant. Contrasting with observations in other nucleic acid-transforming enzymes, these kinetics are strikingly temperature-independent. This indicates (a) nearbarrierless electron transfer reactions and (b) no exchange between different configurations on the timescale up to at least 2 ns, despite the presumed flexibility of Trp214. Results of extensive molecular dynamics simulations are presented to explain this unexpected finding in terms of slowly exchanging protein configurations.
\end{abstract}

Keywords Flavoproteins $\cdot$ Time-resolved fluorescence $\cdot$ Electron transfer $\cdot$ Protein dynamics

\section{Introduction}

Flavins are ubiquitous cofactors found in many proteins involved in the catalysis of electron transfer (ET) and proton transfer reactions. They can be present as the riboflavin (vitamin B2) derivatives flavin mononucleotide (FMN) or flavin adenine dinucleotide (FAD). Flavins are versatile redox partners as they can adopt three different redox states, each with different possible protonation states, overall including five physiologically relevant chemical forms [1]. In the oxidized state FADox, which is the resting state

Pushing the limits of flash photolysis to unravel the secrets of biological electron and proton transfer - a topical issue in honour of Klaus Brettel.

Marten H. Vos

marten.vos@polytechnique.edu

1 LOB, CNRS, INSERM, Ecole Polytechnique, Institut Polytechnique de Paris, 91128 Palaiseau Cedex, France of most flavoproteins, the flavin is intrinsically highly fluorescent, with a fluorescence lifetime of $4-5 \mathrm{~ns}[2,3]$. In proteins, flavin fluorescence is often strongly quenched due to competing photo-induced ET reactions. This is the case in several functionally light-active flavoproteins, such as cryptochrome [4-6] and BLUF domain [7, 8] blue-light sensors, where nearby aromatic amino acids may act as electron donors, and the recently discovered flavoenzyme fatty-acid photodecarboxylase $[9,10]$, where the substrate acts as electron donor, as well as in artificial polypeptideflavin complexes mimicking such functions [undefined]. However, such photochemical processes also frequently occur in the vast majority of flavoproteins whose physiological functioning does not require light absorption. Here, population of the excited state FAD* leads to radical pairs of the form $\mathrm{FAD}^{\bullet-} \mathrm{X}^{\bullet+}$, where $\mathrm{X}$ is a close-by tryptophan or tyrosine residue, from which the charge pairs subsequently recombine, either directly or following further stabilizing electron and/or proton transfer processes [12]. These processes reduce the lifetime of the excited 
state, thus hindering formation of potentially harmful, reactive oxygen species producing long-lived flavin triplet states [13]. In this light, the presence of tryptophan and tyrosine residues in the flavin vicinity can be viewed as photoprotective.

Even in not photocatalytic flavoproteins, characterization of intra-protein flavin photoreduction reactions can be highly informative. First, they can serve to populate and characterize intermediate states that do play roles in physiological reactions, but where they are too short-lived to be detectably accumulated [12, 14, 15]. Second, the kinetic characteristics of the ET reactions are highly sensitive to the configuration of the redox partners and can be used as a probe for the active site [16, undefined], for their heterogeneity as well as for thermal dynamics $[18,19]$. In the simplest view, the ET rate depends on the distance between the reactants' ring systems in an exponentially decreasing manner with an average protein density [20], and barrierless ET can compete with intrinsic fluorescence decay ( $3 \mathrm{~ns})$ for distances up to $\sim 11$ $\AA$ A. Finally, in very recent work, also the spectral characteristics of the flavin and amino acid radical product states have been used to characterize their interaction [21].

TrmFO is a bacterial flavoenzyme that is not photocatalytic, but has recently become a model system for flavin photoreduction $[14,15,22]$. It is involved in post-transcriptional site-specific methylation of tRNA, further using $\mathrm{C}_{2} \mathrm{H}_{4}$ folate as a carbon donor substrate and NADPH as a flavin-reducing substrate [23, 24]. In the available crystal structure, the closest residues to the flavin isoalloxazine ring system are a cysteine and a tyrosine residue (T. thermophilus numbering Cys51 and Tyr343, Fig. 1) [25]. The cysteine residue is thought to be forming a highly fluorescent [26] adduct with the flavin during the reaction cycle [27]. In the absence of the cysteine, the flavin fluorescence is strongly quenched by ET from the close-by ( $3.3 \AA$ A ring-to-ring) tyrosine residue. This feature has allowed to show that tyrosine radicals can be formed and spectroscopically characterized in the visible [15] and infrared [22], in the elusive $\mathrm{TyrOH}^{\bullet+}$ state, which has been invoked as a potential functional intermediate in biochemical reactions $[28,29]$.

In previous work on another nucleotide-transforming bacterial flavoenzyme, thymidylate synthase ThyX, heterogeneous quenching kinetics were used to investigate the conformational dynamics of the active site environment [18], showing a remarkable flexibility of the flavin. In the present work, we extend our work on TrmFO variants along similar lines, by investigating longer range and heterogeneous ET dynamics.

\section{Materials and methods}

Construction of site-directed mutants of TrmFO from Thermus thermophilus and protein expression was performed analogous to the procedures described in Ref. [15]. Experiments were performed with purified proteins suspended in $50 \mathrm{mM}$ Hepes buffer, at $\mathrm{pH} 8.0$, containing up to $250 \mathrm{mM}$ $\mathrm{NaCl}$. All experiments were performed in quartz cells of $1 \mathrm{~mm}$ path length at flavin concentrations in the range of $100-150 \mu \mathrm{M}$. The sample was kept in a thermostattable sample holder. Unless indicated otherwise, the temperature was $20^{\circ} \mathrm{C}$.

Steady state absorption spectra were taken at room temperature using a Shimadzu UV-Vis 1700 spectrometer. Time- and spectrally resolved fluorescence experiments were performed using a Kerr gate-based setup that was described before $[18,30]$. Very briefly, the setup operates at a repetition rate of $500 \mathrm{~Hz}$ and employs excitation pulses centered at $390 \mathrm{~nm}$. The temporal resolution is determined by the Kerr-gate medium, and was either 200 fs (suprasil, used for C51A, C51A/W214F and C51A/W283F mutants)) or $\sim 1$ ps $\left(\mathrm{CS}_{2}\right.$, used for C51A/Y343F and C51A/Y343F/ $\mathrm{W} 214 \mathrm{~F}$ mutants); in the latter case, the signal is higher due to higher efficiency of the medium. Time-resolved absorption experiments were performed as described [15, 31], with pump pulses centered at $390 \mathrm{~nm}$. All time-resolved
Fig. 1 Close-up of the structure of TrmFO from T. thermophilus (PDB ID 3G5Q). Residues critical to this work are shown in orange. The red spring represents the loop consisting of residues 208-222 that is not resolved in the crystal structure

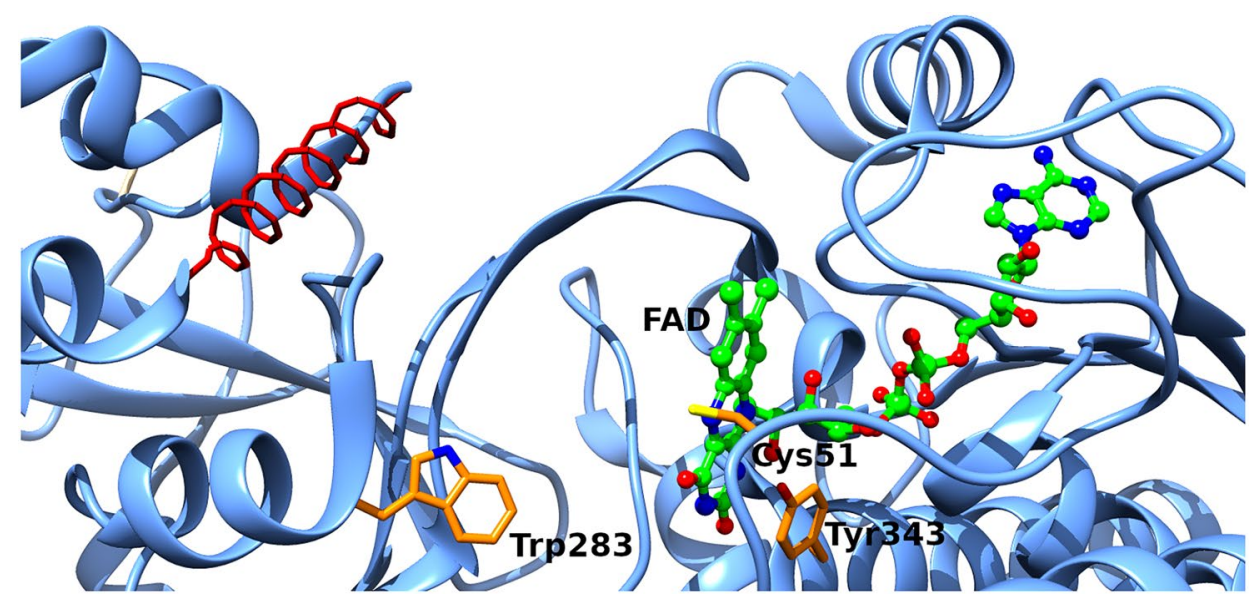


data were globally analyzed in terms of species associated spectra (SAS) using the Glotaran package [32]. For transient absorption data, strong cross phase modulation artifacts around $t=0$ in association with the probe beam spectral dispersion, can lead to modulations of the short timescale SAS as in some traces in Fig. 2c.

For molecular dynamics (MD) simulations, an atomic model of the TrmFO C51A variant was constructed. With the three structures of TrmFO (3G5Q, 3G5R and 3G5S) currently available in the PDB [25] as templates, we used MODELLER [33] to change Cys51 to Ala51 and to construct missing residues of the flexible loop (213-219) and of the C-terminal region (439-443) that are unresolved in all three crystal structures. A set of 10 structures was generated and the best structural model was chosen with Procheck [34]. In addition to crystal waters, a cubic box of water was
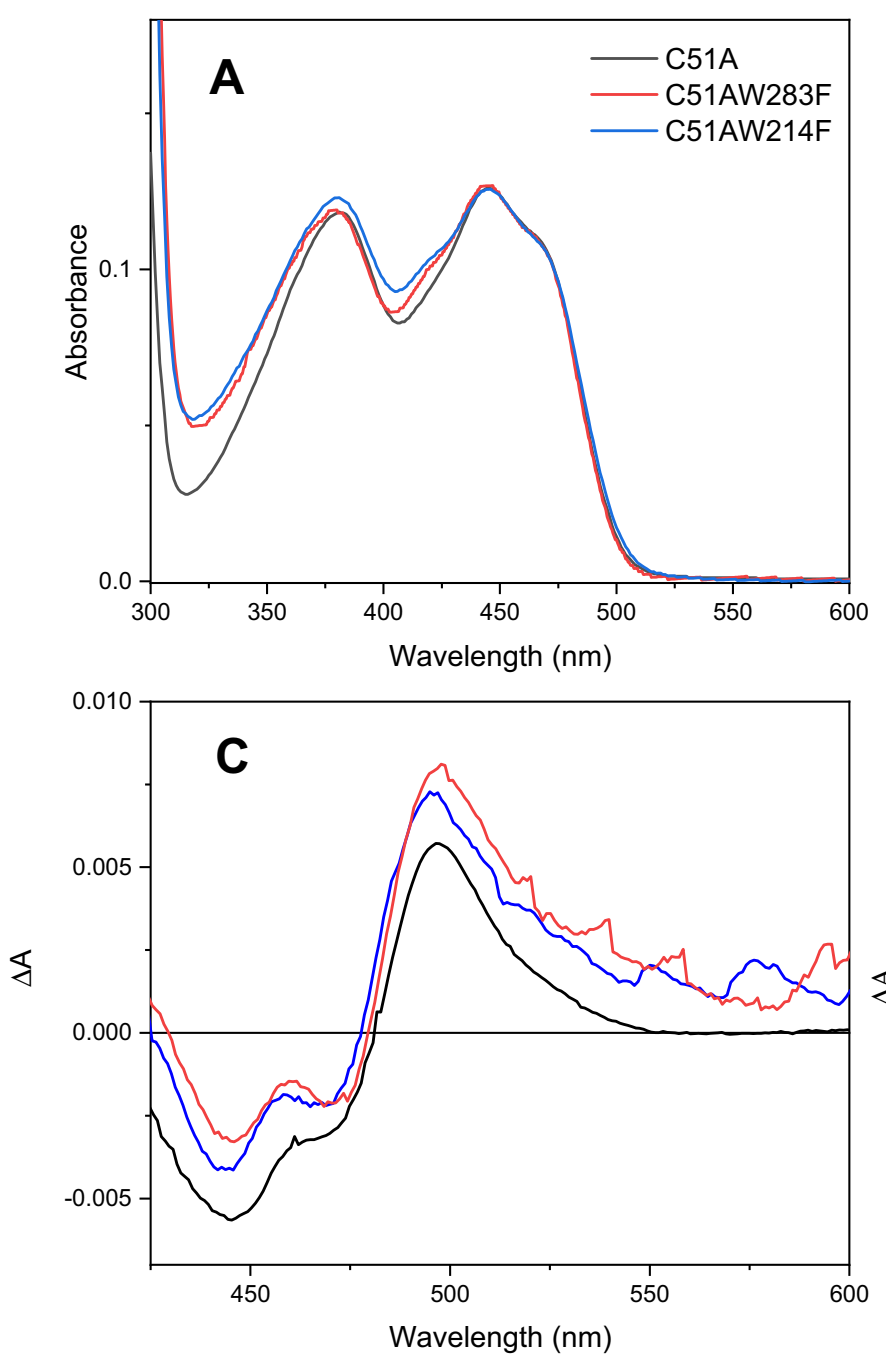

Fig. 2 Photochemical properties of C51A, C51A/W283F and C51A/ W214F TrmFO. a: ground state spectra. b: fluorescence decay at 520 $\mathrm{nm}$. c: species-associated spectra of the $\sim 3 \mathrm{ps}$ decay phase in transient overlaid and waters overlapping the protein and crystal water molecules removed. The size of the water box was chosen so that the shortest distance between protein atoms and the box edges was $20 \AA$. Hydrogen atoms were added using CHARMM [35]. The final system contained a 443 amino acids polypeptidic chain, an FAD cofactor, 27,594 water molecules, and $31 \mathrm{Cl}^{-}$and $36 \mathrm{~K}^{+}$ions corresponding to a $0.15 \mathrm{M}$ ion concentration. Protonation states of histidines were assigned by visual inspection and ideal stereochemistry. Long-range electrostatic interactions were computed using the particle mesh Ewald method [36] and periodic boundary conditions were assumed using a real-space cutoff of $16 \AA$. After energy minimization, the structure was heated from 50 to $300 \mathrm{~K}$, equilibrated at $300 \mathrm{~K}$ for $500 \mathrm{ps}$ and eventually a constant-temperature and pressure 500ns dynamics trajectory was performed with NAMD [37]
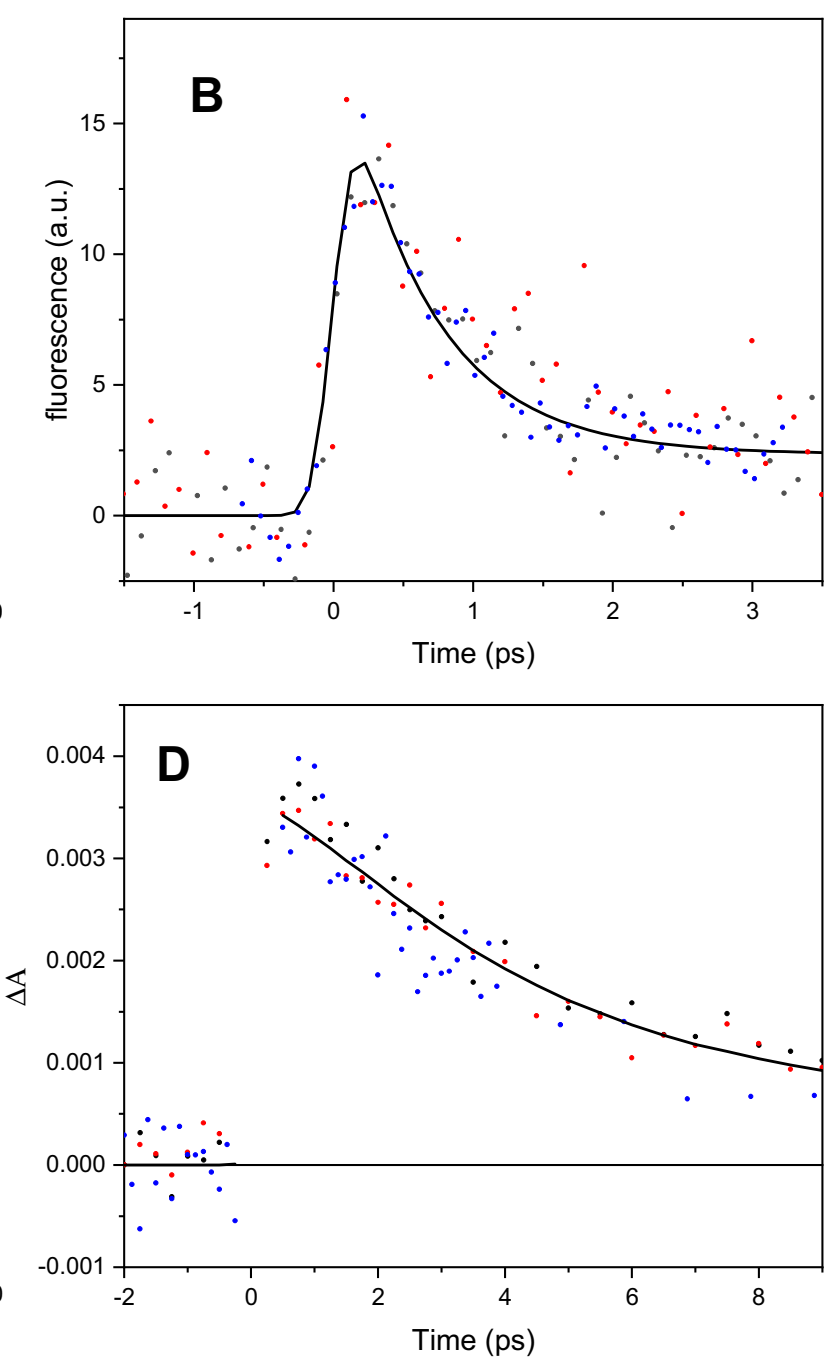

absorption. d: transient absorption at $500 \mathrm{~nm}$. All panels are normalized. Color coding is the same for all panels 
without any restraints. Structures were saved every 1 ps for further analysis. The CHARMM36 force field was used for the protein [38] and the TIP3P model for water [39, 40]. The flavin cofactor was modeled using the force field developed in previous work [41].

Replica Exchange Molecular Dynamics (REMD) [42] simulations of TrmFO C51A were performed using the NAMD software. The model used for these simulations contained the same protein/cofactor complex and was surrounded by 14,534 water molecules and $41 \mathrm{Cl}^{-}$and $46 \mathrm{Na}^{+}$ ions. A number of 16 replicas with a temperature range of 300-330 K was chosen to reach a replica exchange transition probability of about 0.1 [43]. An exchange between replicas was attempted every $1 \mathrm{ps}$ and a $1 \mathrm{fs}$ integration time step was used. Long-range electrostatics were calculated using the Particle-Mesh Ewald summation. Periodic boundary conditions were employed using a rectangular water box with dimensions $94 \AA$, $77 \AA$, and $68 \AA$. A production run of $30 \mathrm{~ns}$ was performed, leading to an ensemble of 480,000 conformations.

\section{Results and discussion}

Previously [15] we showed that Tyr343, located at $3.3 \AA$ from the FAD ring, is the main flavin fluorescence quencher in C51A TrmFO. Other residues that could potentially serve as electron donor to excited flavin are located further away and include the tryptophan residues Trp283 and Trp214. These are respectively located at $8.6 \AA$ in the $T$. thermophilus crystal structure and on a flexible loop that is not resolved in the crystal structure (Fig. 1). These residues are therefore not expected to participate in quenching when Tyr343 is present. This prediction was tested by examining the photochemistry of the C51A/W283F and C51A/W214F mutant proteins.

Figure 2a compares the ground state flavin absorption spectra of these constructs with that of the C51A mutant. The spectra are very similar suggesting that the direct environment of the flavin cofactor is not significantly perturbed by these changes.

Figure $2 \mathrm{~b}$ compares the fluorescence decay of the three constructs. As in the C51A single mutant [15], in both double mutants, the fluorescence predominantly decays with a time constant of $\sim 1 \mathrm{ps}$. This implies that the rate of ET to the flavin is very similar.

Transient absorption experiments on the C51A mutant have shown that following excited state decay a $\mathrm{FAD}^{\bullet-}{ }^{\bullet} \mathrm{TyrOH}^{\bullet+}$ product state is formed that decays back to the ground state by charge recombination in $\sim 3$ ps [15]. In the double mutants, a spectrally very similar product state is formed that also decays in $\sim 3$ ps (Fig. 2c, d). Altogether, these results confirm that Tyr343 is responsible for quenching FAD fluorescence and show that mutation of Trp214 and Trp283 to phenylalanine does not strongly influence the active site conformation.

When Tyr343 is replaced by phenylalanine (C51A/ Y343F mutant) the decay of FAD fluorescence is dramatically slowed ([15], Fig. 3, cf. Figure 2b). Yet, the decay is not fully slowed down to rates expected for unquenched flavin fluorescence $\left((4-5 \mathrm{~ns})^{-1}[2]\right)$. This finding indicates that quenching by other intra-protein electron donors plays a role in this mutant. Notably, the fluorescence decay of the C51A/ $\mathrm{W} 283 \mathrm{~F}$ mutant also is strongly multiphasic; in a multiexponential fit, at least three time constants are required, of $30 \mathrm{ps,}$ $400 \mathrm{ps}$ and $>2 \mathrm{~ns}$. This kinetic heterogeneity suggests that multiple configurations are involved that do not interchange faster than the timescale of the fluorescence decay. Trp214 and Trp283 are among the potential electron donors (see above). In view of the heterogeneity, Trp214 is an interesting candidate, as it is located in a region that is not resolved in the crystal structure and thought to be intrinsically flexible [25], thus potentially adopting different configurations corresponding to different inherent rates of ET to flavin. We therefore constructed the $\mathrm{C} 51 \mathrm{~A} / \mathrm{Y} 283 \mathrm{~F} / \mathrm{W} 214 \mathrm{~F}$ triple mutant and investigated its fluorescence decay. Figure 3 shows that the alteration of Trp214 to redox-inactive phenylalanine further slows down the fluorescence decay, confirming that this tryptophan residue acts as a fluorescence quencher in the C51A/Y283F mutant (without excluding others).

The heterogeneity of the kinetics along with the presumed flexibility of the electron donor environment raises the question whether configurations with significantly different ET characteristics exchange within the picoseconds to early nanoseconds timescale of the fluorescence decays.

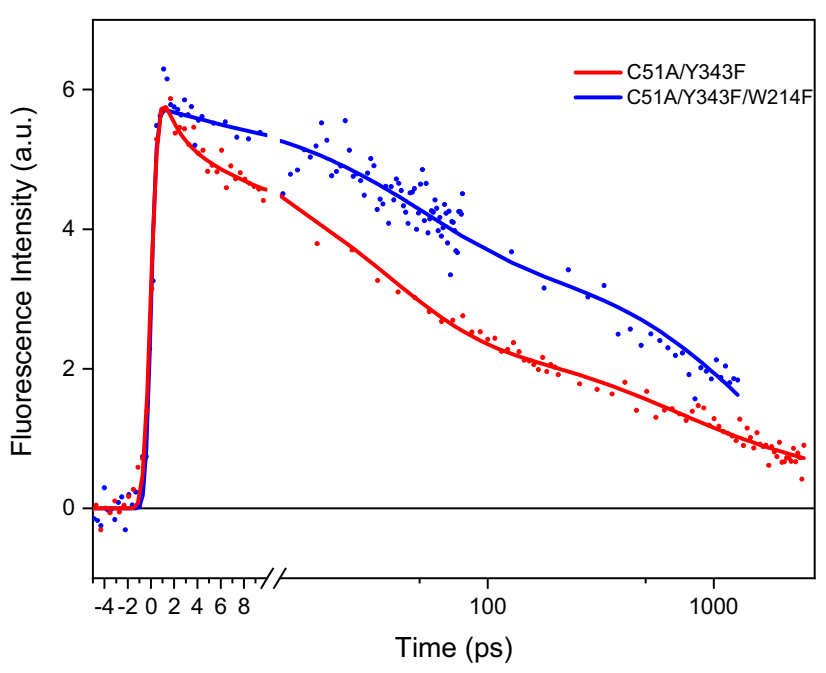

Fig. 3 Effect of mutation of Trp214 to Phe on the fluorescence decay at the 520-nm emission maximum of $\mathrm{C} 51 \mathrm{~A} / \mathrm{Y} 343 \mathrm{~F}$ TrmFO. Time scale is linear up to $10 \mathrm{ps}$ and logarithmic thereafter 
In principle, this issue can be investigated by monitoring the temperature dependence of the fluorescence decay. Such an approach was, for instance, successfully used to demonstrate the extreme flexibility of the active site (and in this case, presumably in particular the flavin cofactor) of thymidylate synthase ThyX from a hyperthermophilic bacterium [18].

Figure 4 shows that the multiphasic fluorescence decay kinetics in C51A/Y343F TrmFO are actually strikingly temperature-independent in the range of $20-40{ }^{\circ} \mathrm{C}$. This observation implies that (1) the intrinsic ET between the electron donor(s) and the flavin takes place in an effectively barrierless regime and (2) exchange between conformations exhibiting significantly different ET rates does not take place on the timescale of the fluorescence decay $(<2 \mathrm{~ns})$. The first assessment is in general agreement with earlier studies on photo-induced ET between flavin-aromatic residue pairs [16] and further validates the interpretation of the temperature dependence of such kinetics in terms of configurational dynamics [18]. Yet, the second assessment was a priori unexpected in view of the above-discussed involvement of Trp214 as electron donor and the presumed location of this residue on a flexible loop.

The proposal that the loop harboring Trp214 is very flexible arises from the observation that this region is not ordered enough to be resolved in the crystal structure [25]. To investigate whether this region is actually disordered in solution, we performed MD simulations of a C51A variant model, based on the wild-type crystal structure (Fig. 1) and an initial loop configuration constructed for the unresolved residues 213-219. To optimally explore the configurational space, REMD simulations were performed in the temperature range $300-330 \mathrm{~K}$, and widely varying configurations

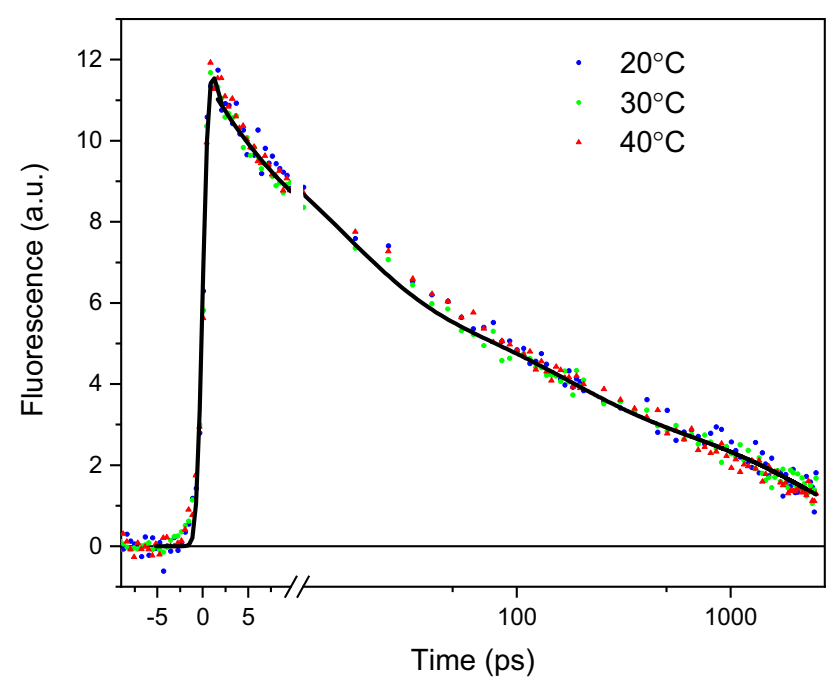

Fig. 4 Temperature dependence of fluorescence decay of C51A/ Y343F TrmFO. Time scale is linear up to $10 \mathrm{ps}$ and logarithmic thereafter were observed, confirming the flexibility of the loop. Figure 5a shows two extreme cases of the range of observed loop conformations; in one case, Trp214 is relatively close to the flavin $(\sim 5 \AA)$, in the other case, it extends well into the solvent. For the first conformation, ET to the flavin is possible within the intrinsic flavin fluorescence decay time; in the other conformation, ET is extremely unlikely due to the large distance between Trp214 and flavin.

A classical MD simulation was performed at a fixed temperature $(300 \mathrm{~K})$ to investigate the dynamics of the flexible loop without accelerating conformational changes as in the REMD simulations presented above. Figure $5 \mathrm{~b}$ shows the closest distance between the rings of the flavin and of Trp214 during the 500-ns trajectory. This distance varies dramatically over this timespan, also fully consistent with the proposed flexibility and macroscopic disorder of the loop. However, the exchange between different configurations occurs mostly on timescales $>10 \mathrm{~ns}$ (spectral density $<0.1 \mathrm{~ns}^{-1}$, Fig. $5 \mathrm{c}$ ), well beyond the window of fluorescence decay. This implies that the configurations, although heterogeneous, are quasi-stable on the 2-ns experimental window of the fluorescence decay. Therefore, the simulations are in agreement with the experimental observations of heterogeneous and strictly temperature-independent fluorescence decay kinetics.

\section{Concluding remarks}

Altogether, the present work has explored the role of potential quenchers of flavin fluorescence in the nucleic-acid transforming enzyme TrmFO from $T$. thermophilus. We show that in the presence of Tyr343, this residue, which closely interacts with the flavin, is virtually the sole effective electron donor to photoexcited flavin. In the absence of this tyrosine, further-lying residues may quench ET, leading consistently to overall slower and also remarkably multiphasic kinetics. The latter property has also been previously observed in the flavoenzyme thymidylate synthase ThyX that, similarly to TrmFO, has to accommodate the relatively large substrates folate, NADPH and nucleotide/nucleic acid [18]. As in ThyX the multiphasic kinetics could be quantitatively analyzed in terms of rapidly $(<2 \mathrm{~ns})$ exchanging ET donor-acceptor configurations, in TrmFO, we focused on the identified Trp214 ET donor, located on a flexible loop. As discussed above, the properties of the decay kinetics imply that the Trp214-flavin pairs, although heterogeneous, do not rapidly exchange configurations. Subsequent molecular dynamics simulations indeed indicate that the region containing Trp214 is highly flexible, as suggested by the crystal structure [25], but can be considered quasi-static on the timescale of fluorescence decay. This regime of conformational fluctuation may be functionally relevant as it has been 

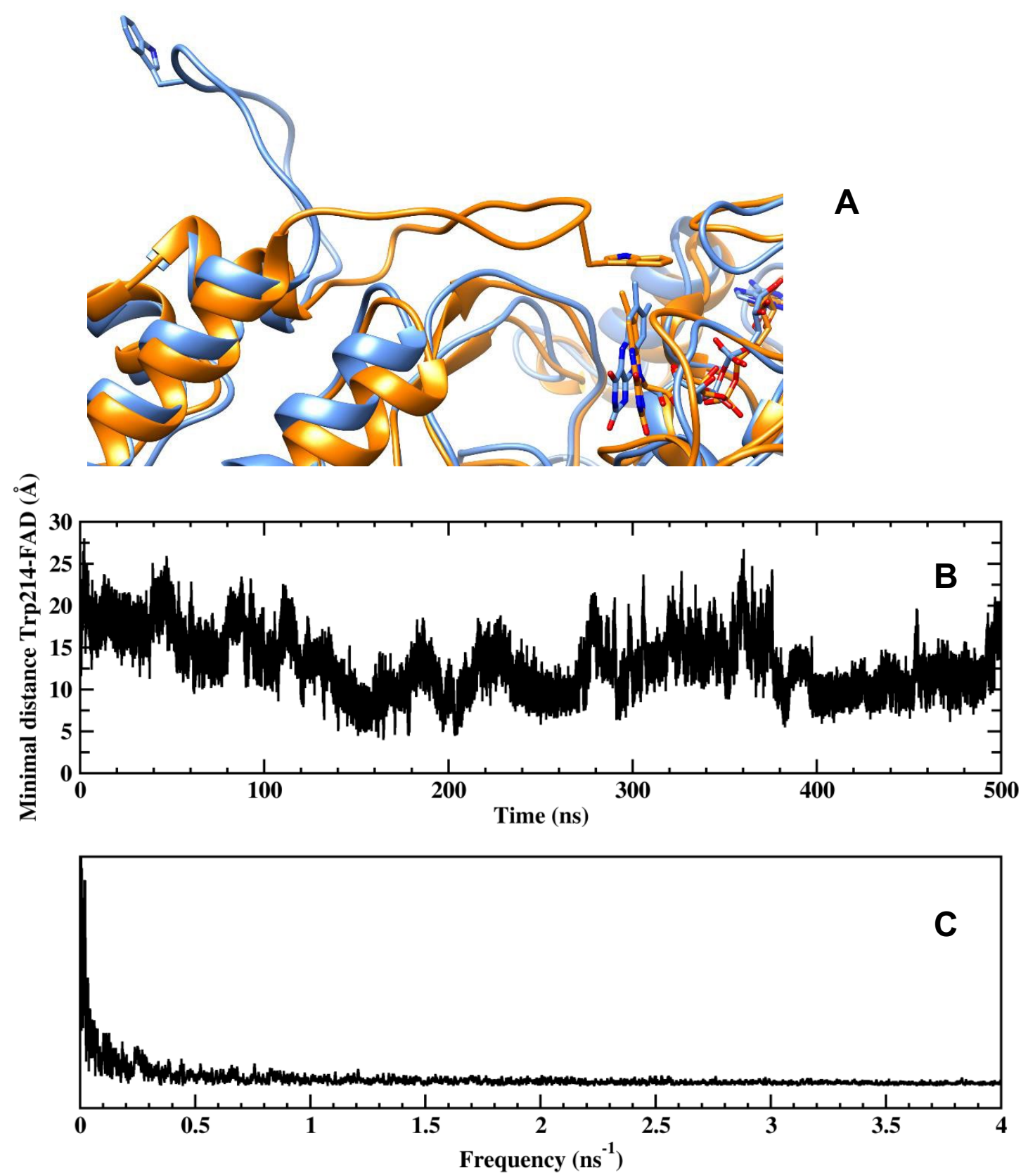

Fig. 5 MD simulations of C51A TrmFO. a: two of the configurations of the loop containing Trp214 observed during 300-330 K REMD simulations. FAD and Trp214 are shown as lines; (b): The shortest

suggested that the rearrangement of this loop is involved in tRNA substrate binding [25]. The comparison between the ThyX and TrmFO protein systems illustrates the power of the employed photochemical technique to experimentally discern regimes of protein dynamics, even in non-naturally photoactive flavoproteins. In future work, we foresee to further explore the role in the quenching dynamics of TrmFO distance between atoms of the Trp214 and isoalloxazine ring systems during a 500-ns MD simulation performed at $300 \mathrm{~K}$. c: Fourier transform spectrum of the distance in B3

of other potential quenchers, including Trp283, which has been suggested to interact with the tRNA substrate [23, 25].

Acknowledgements B. Z. is supported by a China Scholarship Council $\mathrm{PhD}$ scholarship. Part of this work was performed using High Performance Computing resources from Grand Equipement National pour le Calcul Intensif [Centre de Calcul Recherche et Technologie/ Centre Informatique National de l'Enseignement Supérieur/Institut du 
Développement et des Ressources en Informatique Scientifique] (Grant 2018-2019, project number A0020706913).

Data availability Not applicable.

Code availability Not applicable.

\section{Declarations}

Conflict of interest Not applicable.

\section{References}

1. Miura, R. (2001). Versatility and specificity in flavoenzymes: control mechanisms of flavin reactivity. Chemical Record, 1(3), 183-194. https://doi.org/10.1002/tcr.1007

2. van den Berg, P. A. W., Feenstra, K. A., Mark, A. E., Berendsen, H. J. C., \& Visser, A. J. W. G. (2002). Dynamic conformations of flavin adenine dinucleotide: simulated molecular dynamics of the flavin cofactor related to the time-resolved fluorescence characteristics. The Journal of Physical Chemistry B, 106(34), 8858-8869. https://doi.org/10.1021/jp020356s

3. Brazard, J., Usman, A., Lacombat, F., Ley, C., Martin, M. M., \& Plaza, P. (2011). New insights into the ultrafast photophysics of oxidized and reduced FAD in solution. Journal of Physical Chemistry A, 115(15), 3251-3262. https://doi.org/10.1021/jp110 $741 \mathrm{y}$

4. Aubert, C., Mathis, P., Eker, A. P. M., \& Brettel, K. (1999). Intraprotein electron transfer between tyrosine and tryptophan in DNA photolyase from Anacystis nidulans. Proceedings of the National academy of Sciences of the United States of America, 96(10), 5423-5427

5. Giovani, B., Byrdin, M., Ahmad, M., \& Brettel, K. (2003). Lightinduced electron transfer in a cryptochrome blue-light photoreceptor. Natural Structural Biology, 10(6), 489-490

6. Lacombat, F., Espagne, A., Dozova, N., Plaza, P., Muller, P., Brettel, K., et al. (2019). Ultrafast oxidation of a tyrosine by protoncoupled electron transfer promotes light activation of an animallike cryptochrome. Journal of the American Chemical Society, 141(34), 13394-13409. https://doi.org/10.1021/jacs.9b03680

7. Lukacs, A., Brust, R., Haigney, A., Laptenok, S. P., Addison, K., Gil, A., et al. (2014). BLUF domain function does not require a metastable radical intermediate state. Journal of the American Chemical Society, 136(12), 4605-4615. https://doi.org/10.1021/ ja4121082

8. Mathes, T., van Stokkum, I. H., Kennis, J. T. (2014). Photoactivation mechanisms of flavin-binding photoreceptors revealed through ultrafast spectroscopy and global analysis methods. In Weber, S., Schleicher, E. (Eds.), Flavins and Flavoproteins: Methods and Protocols. Springer

9. Sorigué, D., Légeret, B., Cuiné, S., Blangy, S., Moulin, S., Billon, E., et al. (2017). An algal photoenzyme converts fatty acids to hydrocarbons. Science, 357(6354), 903-907. https://doi.org/10. 1126/science.aan6349

10. Sorigué, D., Hadjidemetriou, K., Blangy, S., Gotthard, G., Bonvalet, A., Coquelle, N., et al. (2021). Mechanism and dynamics of fatty acid photodecarboxylase. Science, 372, eabd5687

11. Bialas, C., Barnard, D. T., Auman, D. B., Mcbride, R. A., Jarocha, L. E., Hore, P. J., et al. (2019). Ultrafast flavin/tryptophan radical pair kinetics in a magnetically sensitive artificial protein. Physical Chemistry, 21(25), 13453-13461

12. Nag, L., Lukacs, A., \& Vos, M. H. (2019). Short-lived radical intermediates in the photochemistry of glucose oxidase.
ChemPhysChem, 20(14), 1793-1798. https://doi.org/10.1002/ cphc.201900329

13. Ernst, S., Rovida, S., Mattevi, A., Fetzner, S., \& Drees, S. L. (2020). Photoinduced monooxygenation involving NAD(P) H-FAD sequential single-electron transfer. Nature Comm, 11(1), 2600. https://doi.org/10.1038/s41467-020-16450-y

14. Dozova, N., Lacombat, F., Bou-Nader, C., Hamdane, D., \& Plaza, P. (2019). Ultrafast photoinduced flavin dynamics in the unusual active site of the tRNA methyltransferase TrmFO. Physical Chemistry Chemical Physics: PCCP, 21(17), 8743-8756. https://doi.org/10.1039/c8cp06072j

15. Nag, L., Sournia, P., Myllykallio, H., Liebl, U., \& Vos, M. H. (2017). Identification of the $\mathrm{TyrOH}^{\bullet+}$ radical cation in the flavoenzyme TrmFO. Journal of the American Chemical Society, 139(33), 11500-11505. https://doi.org/10.1021/jacs.7b04586

16. Mataga, N., Chosrowjan, H., Shibata, Y., Tanaka, F., Nishina, Y., \& Shiga, K. (2000). Dynamics and mechanisms of ultrafast fluorescence quenching reactions of flavin chromophores in protein nanospace. The Journal of Physical Chemistry B, 104(45), 10667-10677

17. Zhong, D., \& Zewail, A. H. (2001). Femtosecond dynamics of flavoproteins: charge separation and recombination in riboflavin (vitamin B2)-binding protein and in glucose oxidase enzyme. Proceedings of the National academy of Sciences of the United States of America, 98, 11867-11872

18. Laptenok, S. P., Bouzhir-Sima, L., Lambry, J.-C., Myllykallio, H., Liebl, U., \& Vos, M. H. (2013). Ultrafast real time visualization of the active site flexibility of the flavoenzyme thymidylate synthase ThyX. Proceedings of the National academy of Sciences of the United States of America, 110, 8924-8929

19. Yang, H., Luo, G., Karnchanaphanurach, P., Louie, T.-M., Rech, I., Cova, S., et al. (2003). Protein conformational dynamics probed by single-molecule electron transfer. Science, 302(5643), 262-266. https://doi.org/10.1126/science.1086911

20. Moser, C. C., Anderson, J. L. R., \& Dutton, P. L. (2010). Guidelines for tunneling in enzymes. Biochimica et Biophysica Acta, 1797(9), 1573-1586

21. Zhuang, B., Seo, D., Aleksandrov, A., \& Vos, M. H. (2021). Characterization of light-induced short-lived interacting radicals in the active site of flavoprotein ferredoxin-NADP+ oxidoreductase. Journal of the American Chemical Society, 143, 2457-2768

22. Pirisi, K., Nag, L., Fekete, Z., Iuliano, J. N., Tollentino Collado, J., Clark, I. P., et al. (2021). Identification of the vibrational marker of tyrosine cation radical using ultrafast transient infrared spectroscopy of flavoprotein systems. Photochemical and Photobiological Sciences, 20, 369-378

23. Hamdane, D., Argentini, M., Cornu, D., Myllykallio, H., Skouloubris, S., Hui-Bon-Hoa, G., et al. (2011). Insights into Folate/FADdependent tRNA methyltransferase mechanism: role of two highly conserved cysteines in catalysis. Journal of Biological Chemistry, 286(42), 36268-36280. https://doi.org/10.1074/jbc.M111.256966

24. Urbonavicius, J., Skouloubris, S., Myllykallio, H., \& Grosjean, H. (2005). Identification of a novel gene encoding a flavin-dependent tRNA: ${ }^{5} U$ methyltransferase in bacteria -evolutionary implications. Nucleic Acids Research, 33(13), 3955-3964

25. Nishimasu, H., Ishitani, R., Yamashita, K., Iwashita, C., Hirata, A., Hori, H., et al. (2009). Atomic structure of a folate/FADdependent tRNA T54 methyltransferase. Proceedings of the National academy of Sciences of the United States of America, 106(20), 8180-8185. https://doi.org/10.1073/pnas.0901330106

26. Sournia, P. (2016). La méthylation flavine-dépendante d'acides nucléiques : aspects évolutifs, métaboliques, biochimiques et spectroscopiques. Ecole Polytechnique.

27. Hamdane, D., Bruch, E., Un, S., Field, M., \& Fontecave, M. (2013). Activation of a unique flavin-dependent tRNA-methylating 
agent. Biochemistry, 52(49), 8949-8956. https://doi.org/10.1021/ bi4013879

28. Renger, G. (2013). Apparatus and mechanism of photosynthetic water splitting as nature's blueprint for efficient solar energy exploitation. In R. Razeghifard (Ed.), Natural and artificial photosynthesis.John Wiley and Son.

29. Gauden, M., van Stokkum, I. H. M., Key, J. M., Lührs, D. C., van Grondelle, R., Hegemann, P., et al. (2006). Hydrogen-bond switching through a radical pair mechanism in a flavin-binding photoreceptor. Proceedings of the National academy of Sciences of the United States of America, 103(29), 10895-10900. https:// doi.org/10.1073/pnas.0600720103

30. Laptenok, S. P., Nuernberger, P., Lukacs, A., \& Vos, M. H. (2014). Subpicosecond Kerr-gate spectrofluorometry. In Y. Engelborghs \& A. J. W. G. Visser (Eds.), Methods in molecular biology, fluorescence spectroscopy and microscopy: methods and protocols.Humana Press.

31. Lambry, J.-C., Stranava, M., Lobato, L., Martinkova, M., Shimizu, T., Liebl, U., et al. (2016). Ultrafast spectroscopy evidence for picosecond ligand exchange at the binding site of a heme protein: heme-based sensor YddV. Journal of Physical Chemistry Letters, 7(1), 69-74. https://doi.org/10.1021/acs.jpclett.5b02517

32. Snellenburg, J. J., Laptenok, S. P., Seger, R., Mullen, K. M., \& van Stokkum, I. H. M. (2012). Glotaran: a java-based graphical user interface for the R package TIMP. J Stat Software, 49(3), 2

33. Webb, B., \& Sali, A. (2016). Comparative protein structure modeling using modeller. Curr Protoc Bioinform, 54(1), 561-5637. https://doi.org/10.1002/cpbi.3

34. Laskowski, R. A., MacArthur, M. W., Moss, D. S., \& Thornton, J. M. (1993). PROCHECK: a program to check the stereochemical quality of protein structures. Journal of Applied Crystallography, 26(2), 283-291. https://doi.org/10.1107/S0021889892009944
35. Brooks, B. R., Brooks, C. L., Mackerell, A. D., Nilsson, L., Petrella, R. J., Roux, B., et al. (2009). CHARMM: the biomolecular simulation program. J Comp Chem, 30(10), 1545-1614

36. Darden, T., York, D., \& Pedersen, L. (1993). Particle mesh Ewald: An $\mathrm{N} \cdot \log (\mathrm{N})$ method for Ewald sums in large systems. The Journal of Chemical Physics, 98(12), 10089-10092. https://doi.org/ 10.1063/1.464397

37. Phillips, J. C., Braun, R., Wang, W., Gumbart, J., Tajkhorshid, E., Villa, E., et al. (2005). Scalable molecular dynamics with NAMD. J Comp Chem, 26(16), 1781-1802. https://doi.org/10.1002/jcc. 20289

38. Huang, J., \& MacKerell, A. D., Jr. (2013). CHARMM36 all-atom additive protein force field: validation based on comparison to NMR data. J Comp Chem, 34(25), 2135-2145. https://doi.org/ $10.1002 /$ jcc. 23354

39. Jorgensen, W. L., Chandrasekhar, J., Madura, J. D., Impey, R. W., \& Klein, M. L. (1983). Comparison of simple potential functions for simulating liquid water. The Journal of Chemical Physics, 79(2), 926-935. https://doi.org/10.1063/1.445869

40. Neria, E., Fischer, S., \& Karplus, M. (1996). Simulation of activation free energies in molecular systems. The Journal of Chemical Physics, 105(5), 1902-1921. https://doi.org/10.1063/1.472061

41. Aleksandrov, A. (2019). A molecular mechanics model for Flavins. J Comp Chem, 40(32), 2834-2842. https://doi.org/10.1002/ jcc. 26061

42. Sugita, Y., \& Okamoto, Y. (1999). Replica-exchange molecular dynamics method for protein folding. Chemical Physics Letters, 314(1), 141-151. https://doi.org/10.1016/S0009-2614(99)01123-9

43. Patriksson, A., \& van Spoel, D. (2008). A temperature predictor for parallel tempering simulations. Physical Chemistry Chemical Physics: PCCP, 10(15), 2073-2077. https://doi.org/10.1039/ B716554D 